\title{
Partitioning of copy-number genotypes in pedigrees
}

\author{
Louis-Philippe Lemieux Perreault*1,2, Gregor U Andelfinger2,3, Géraldine Asselin¹ and Marie-Pierre Dubé*1,2
}

\begin{abstract}
Background: Copy number variations (CNVs) and polymorphisms (CNPs) have only recently gained the genetic community's attention. Conservative estimates have shown that CNVs and CNPs might affect more than $10 \%$ of the genome and that they may be at least as important as single nucleotide polymorphisms in assessing human variability. Widely used tools for CNP analysis have been implemented in Birdsuite and PLINK for the purpose of conducting genetic association studies based on the unpartitioned total number of CNP copies provided by the intensities from Affymetrix's Genome-Wide Human SNP Array. Here, we are interested in partitioning copy number variations and polymorphisms in extended pedigrees for the purpose of linkage analysis on familial data.

Results: We have developed CNGen, a new software for the partitioning of copy number polymorphism using the integrated genotypes from Birdsuite with the Affymetrix platform. The algorithm applied to familial trios or extended pedigrees can produce partitioned copy number genotypes with distinct parental alleles. We have validated the algorithm using simulations on a complex pedigree structure using frequencies calculated from a real dataset of 300 genotyped samples from 42 pedigrees segregating a congenital heart defect phenotype.

Conclusions: CNGen is the first published software for the partitioning of copy number genotypes in pedigrees, making possible the use CNPs and CNVs for linkage analysis. It was implemented with the Python interpreter version 2.5.2. It was successfully tested on current Linux, Windows and Mac OS workstations.
\end{abstract}

\section{Background}

Copy number variations (CNVs) and polymorphisms (CNPs) have recently gained much interest as a novel tool to study the relationship between genomic variation and disease. CNVs and CNPs are widespread throughout the genome and were shown to be largely heritable while being responsible for a significant amount of inter-variability in human [1]. They can also appear de novo at a significant rate, both in germline and somatic cells [2]. Any variation in copy number has the possibility of affecting a wide spectrum of genes, which might lead to genomic disorders [3]. Variation in gene-expression levels can occur for genes located within a region of copy number variation [2], and negative correlations between $\mathrm{CNV}$ and gene expression were reported in approximately $10 \%$

\footnotetext{
*Correspondence: louis-philippe.lemieux.perreault@umontreal.ca, mariepierre.dube@umontreal.ca

${ }^{1}$ Montreal Heart Institute Research Center, 500, Bélanger Street, Montréal, Canada

Full list of author information is available at the end of the article
}

of cases [4]. It is currently estimated that up to $12 \%$ of the genome is subject to copy number variations $[5,6]$. Those genetic variations are likely to play an important role in the etiology of common disease and sporadic birth defects [1], partly attributable to their higher mutation rate as compared to point mutation [7] and due to their considerable genomic coverage.

High-density SNP genotyping arrays are commonly used for CNV/CNP analysis. Those arrays provide signal intensities of alleles across all SNPs which can be used to infer copy numbers along with a selection of CNV-specific probes. The presence of a CNV/CNP region has the potential to confuse SNP calling algorithms if unaccounted for, as SNPs can be represent with multiple or single alleles. It is then crucial to gain knowledge of CNV and CNP in genetic analysis, even when using SNPs as a marker.

While amenable to genetic association studies, the use of CNVs and CNPs in linkage analysis with multi-generational family data has up to now been greatly limited by the requirement of chromosome-specific copy number 
assignments, which, to our knowledge, none of the current software indexed in the literature is able to provide. Multi-allelic partitioned copy number polymorphisms have the potential to offer a new and powerful tool for linkage analysis. Today's high density SNP panels offer near-optimal coverage for linkage analysis. However, some regions, especially those with copy number polymorphisms, may have been less well covered due to the requirements of Mendelian consistency prior to linkage analysis. Although representing only a minute fraction of the genome, the partitioning of copy number genotypes has the potential to help fill-in the remaining linkage coverage gaps.

The use of genome-wide association studies (GWAS) with unrelated cases and controls is a popular approach for the discovery of genetic variants responsible for common genetic diseases [8]. Linkage analysis with extended pedigrees is of limited use for the identification of common polymorphisms of low effect, but it does offer high detection power with more penetrant variants even in the presence of multiple rare causal variants at a single locus [9] or highly penetrant rare variants throughout the genome. Furthermore, the combined use of pedigreebased linkage analysis and association studies in a multistage approach was argued by Elston et al. to be both powerful and advantageous [9]. Significantly linked markers can emphasize candidate genes for subsequent association study and information on candidate loci can be incorporated into association tests using either a generalized logistic regression [10] or a quantitative linkage score [11].

Here, we are interested in using CNV and CNP data from the Affymetrix 6.0 chip analyzed with the Fawkes program of the Birdsuite software [12,13]. Fawkes creates an integrated genotype from SNPs, rare copy number variations and common copy number polymorphisms genotypes information, providing the number and type ( $A$ or $B$ ) of each allele for each SNP on the Affymetrix Genome-Wide Human SNP Array 5.0 and 6.0 chips. While the suite comes with Python scripts for file compatibility with the whole-genome association toolset PLINK [14], no software is available to conduct chromosome assignment of the copy number genotypes based on pedigree information. We propose a new algorithm called CNGen that uses SNP genotypes in multi-generational pedigrees to convert Fawkes' genotypes into partitioned copy number genotypes ( $\mathrm{CN}$ genotypes) which can then be treated as multi-allelic markers by common linkage software such as MERLIN [15]. We have developed Python scripts to encode CN genotypes into multi-allelic genotypes. We have validated and successfully applied the algorithm in the analysis of multi-generational pedigrees through simulation procedures.

\section{Implementation}

The standard Fawkes output file is tabulated with samples in columns and probe sets (SNPs) in rows. Each cell contains a Fawkes call that is a comma-separated value of the form $[a, b]$ where $a$ is the number of copies of allele $A$ and $b$, the number of copies of allele $B$. Five different Fawkes calls are possible:

1. undefined calls, from a probe set of the form $[-1,-1]$ that was unresolved by Fawkes;

2. heterozygous calls of the form $[a, b]$ where $a$ and $b$ $\neq 0$;

3 . null calls of the form $[0,0]$, representing a null genotype;

4. hemizygous calls of the form $[0, b]$ or $[a, 0]$, where $a$ and $b=1$;

5. homozygous calls, of the form $[0, b]$ or $[a, 0]$, where $a$ and $b>1$.

CNGen converts Fawkes calls into partitioned CN genotypes as comma-separated values of the form $\left[T_{1} m, T_{2} n\right]$ where $T_{i}$ is the allele type (one of $A, B$ or $N$ for null) on one of the parental chromosome, and $m$ and $n$ represent the number of copies of the named allele type on the specified chromosome. The $N$ allele type represents an absence of either an $A$ or $B$ allele on a given parental chromosome. The partitioning of copy numbers is accomplished according to the rules of Mendelian transmission and under the general assumption that ancestral copy number expansions were of the same allele type, i.e. a copy number expansion from 1 to 2 copies is not allowed to bear both $A$ and $B$ alleles on the same chromosome strand.

This last assumption affects only copy numbers of two or more, since single-copy alleles will result in one copy which will by default be located on a single chromosome. Situations with two copies where the true CN genotype is $[A 2, N],[B 2, N]$ and $[A 1, B 1]$ will be appropriately called. However true $[A 1 B 1, N]$ will not and will likely give rise to Mendelian inconsistencies which will be coded as undefined by the CNGen algorithm. Expansions beyond 2 copies were found less frequently than 0,1 , and 2 copies by a survey of 300 genotyped individuals in 42 pedigrees presenting a congenital heart defect phenotype. Overall, only $0.07 \%$ of Fawkes calls had three or more copies (expansions), compared to $2.2 \%$ with 0 or 1 copy (deletions) and $97.6 \%$ with 2 copies, with the rest being undefined Fawkes calls.

\section{Step 1 - Partitioning of non-homozygous calls}

The algorithm begins by parsing the Fawkes calls to generate in this first pass the $\mathrm{CN}$ genotypes for the first four of the five possible Fawkes calls. Undefined and null Fawkes genotypes are set to undefined or null CN genotypes, respectively. For single hemizygous Fawkes genotype, the first chromosome is set to hold the deletion $(N)$ 
Table 1: Direct Fawkes conversion. Example of direct conversion from integrated genotypes (Fawkes to CN genotypes. The type-1 homozygous genotypes are converted using information from first-degree relatives with one of those Fawkes calls: heterozygous, null or hemizygous.

\begin{tabular}{ccc}
\hline Fawkes genotypes & Undefined & CN genotypes \\
\hline$-1,-1$ & $\rightarrow$ & $-1,-1$ \\
& & \\
$a, b(a$ and $b \neq 0)$ & Heterozygous & $A a, B b$ \\
& $\rightarrow$ & $N, N$ \\
0,0 & Null \\
& $\rightarrow$ & \\
\hline & & \\
\hline & & \\
0,1 & Single hemizygous & $B 1, N$ \\
\hline
\end{tabular}

and the other, the given allele $(A$ or $B)$. Finally, heterozygous Fawkes calls are partitioned such that each chromosome receives the copies of only one allele type. Those conversions from Fawkes genotypes to partitioned $\mathrm{CN}$ genotypes are summarized in Table 1.

\section{Step 2 - Partitioning of type-I homozygous calls}

We distinguish two types of homozygous Fawkes calls based on the genotype conversion method used: type-I and type-II. CN genotype partitioning for type-I homozygous Fawkes calls is solved by relying on information from a heterozygous first-degree relative and assuming Mendelian transmission. The algorithm searches for heterozygous first-degree relatives (parents, children and siblings) of the index individual to be converted (I), as those will have partitioned $\mathrm{CN}$ genotypes that can be used as reference. Figure 1 presents the different scenarios for type-I homozygote partitioning.

\section{Step $2 a$}

When a parent of $I$ has a heterozygous $\mathrm{CN}$ genotype of the form $\left[A m_{p}, B n_{p}\right]$, then $I$ is assigned the following $\mathrm{CN}$ genotype:

$$
I_{C N, L}= \begin{cases}{\left[A m_{p}, A a-m_{p}\right]} & \text { if } I_{F, L}=[a, 0] \\ {\left[B n_{p}, B b-n_{p}\right]} & \text { if } I_{F, L}=[0, b]\end{cases}
$$

where $I_{F, L}$ and $I_{C N, L}$ are the Fawkes genotype and the new $\mathrm{CN}$ genotype of the index individual at locus $L$, respectively (Figure $1 \mathrm{~A}$ ). If $a-m_{p}$ or $b-n_{p}$ equals 0 , the second partitioned $\mathrm{CN}$ allele becomes $N$.

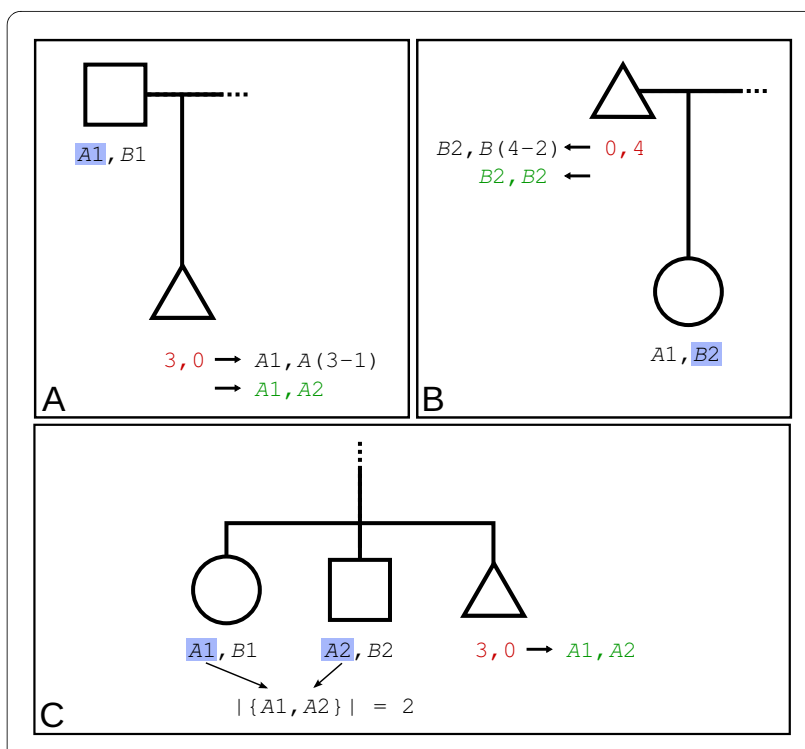

Figure 1 Type-I homozygous conversion. Representation of the conversion of Type-l homozygous Fawkes genotypes (red) into partitioned CN genotypes (green). The triangle represents the index individual (I). (A) Conversion of I's Fawkes genotype using one heterozygous parent, (B) one heterozygous child or (C) two heterozygous siblings. In each case, the sex of the individual used for conversion is not important.

\section{Step $2 b$}

If $I$ does not have a heterozygous parent, the algorithm searches for the presence of a child with a heterozygous $\mathrm{CN}$ genotype of the form $\left[A m_{c}, B n_{c}\right]$. The partition of the $\mathrm{CN}$ genotype is solved as presented in Equation (1) by replacing $m_{p}$ and $n_{p}$ by $m_{c}$ and $n_{c}$ respectively (Figure $1 \mathrm{~B}$ ).

\section{Step 2c}

If $I$ does not have a heterozygous child, then the algorithm searches for the presence of two siblings with distinct heterozygous $\mathrm{CN}$ genotypes $\left[A m_{s 1}, B n_{s 1}\right]$ and $\left[A m_{s 2}\right.$, $B n_{s 2}$ ] for which the cardinality of the pool of $\mathrm{CN}$ alleles of the same type as $I$ is two, i.e. $\left(\left|\left\{A m_{s 1}, A m_{s 2}\right\}\right|=2\right.$ if $I_{F, L}=$ $\left.[a, 0], m_{s 1} \neq m_{s 2}\right)$ or $\left(\left|\left\{B n_{s 1}, B n_{s 2}\right\}\right|=2\right.$ if $I_{F, L}=[0, b], n_{s 1} \neq$ $\left.n_{s 2}\right)$. Then, $I$ is assigned the following $C N$ genotype:

$$
I_{C N, L}= \begin{cases}{\left[A m_{s 1}, A m_{s 2}\right]} & \text { if } I_{F, L}=\left[m_{s 1}+m_{s 2}, 0\right] \\ {\left[B n_{s 1}, B n_{s 2}\right]} & \text { if } I_{F, L}=\left[0, n_{s 1}+n_{s 2}\right] \\ \text { do nothing } & \text { otherwise }\end{cases}
$$

Restricting the conditions $m_{s 1} \neq m_{s 2}$ or $n_{s 1} \neq n_{s 2}$, ensures that both $\mathrm{CN}$ alleles originate from the two distinct parents (Figure 1C). Any Fawkes homozygous calls that remain un-converted are then flagged as type-2 Fawkes homozygous calls and the algorithm proceeds to step 3. 


\section{Step 3 - Partitioning of type-II homozygous calls}

$\mathrm{CN}$ genotype partitioning of type-II homozygous Fawkes calls proceeds by assuming Mendelian transmission of $\mathrm{CN}$ alleles and by relying on information in the nuclear pedigree of $I$. The algorithm searches for a solution according to the following sequential attempts. Figure 2 presents the different scenarios for type-II homozygous partitioning.

\section{Step $3 a$}

First, the algorithm searches for the presence of one parent of $I$ that is homozygous for a CN genotype of the same allele-type as $I$ such as $\left[T_{1} m_{p}, T_{2} n_{p}\right]$ where $T_{1}=T_{2}$, $T_{i=1,2} \quad\{A, B, N\}$ and $m_{p}=n_{p}$; in which case $I$ is assigned its $\mathrm{CN}$ genotype according to Equation (1) (Figure 2A).

\section{Step $3 b$}

Analogously to step $3 \mathrm{a}$ above, a child of $I$ presenting a homozygous $\mathrm{CN}$ genotype $\left[T_{1} m_{c}, T_{2} n_{c}\right]$ can be used according to Equation (1) $\left(m_{c}\right.$ replacing $m_{p}$ and $\left.n_{c}, n_{p}\right)$ (Figure 2B).

\section{Step $3 c$}

If no such parent or child exists, the algorithm then searches for the presence of two siblings, one with a heterozygous genotype of the form $\left[A m_{s 1}, B n_{s 1}\right]$, and the other one with a homozygous $\mathrm{CN}$ genotype with identical $\mathrm{CN}$ alleles excluding null alleles and of a different alleletype than that of $I$ (i.e. $\left[A m_{s 2}, A n_{s 2}\right]$ if I's Fawkes genotypes is $[0, b]$ or $\left[B m_{s 2}, B n_{s 2}\right]$ if $I$ s Fawkes genotypes is $\left.[a, 0]\right)$. In this case, $I_{C N, L}$ is defined by Equation (1) with replacement of $m_{p}$ and $n_{p}$ by $m_{s 1}$ and $n_{s 1}$, respectively (Figure $2 \mathrm{C}$ ).

Step 3d

If I's genotype at the given locus remains unconverted, the algorithm searches for the presence of one child and the spouse of $I$ where both have distinct $\mathrm{CN}$ genotypes. In

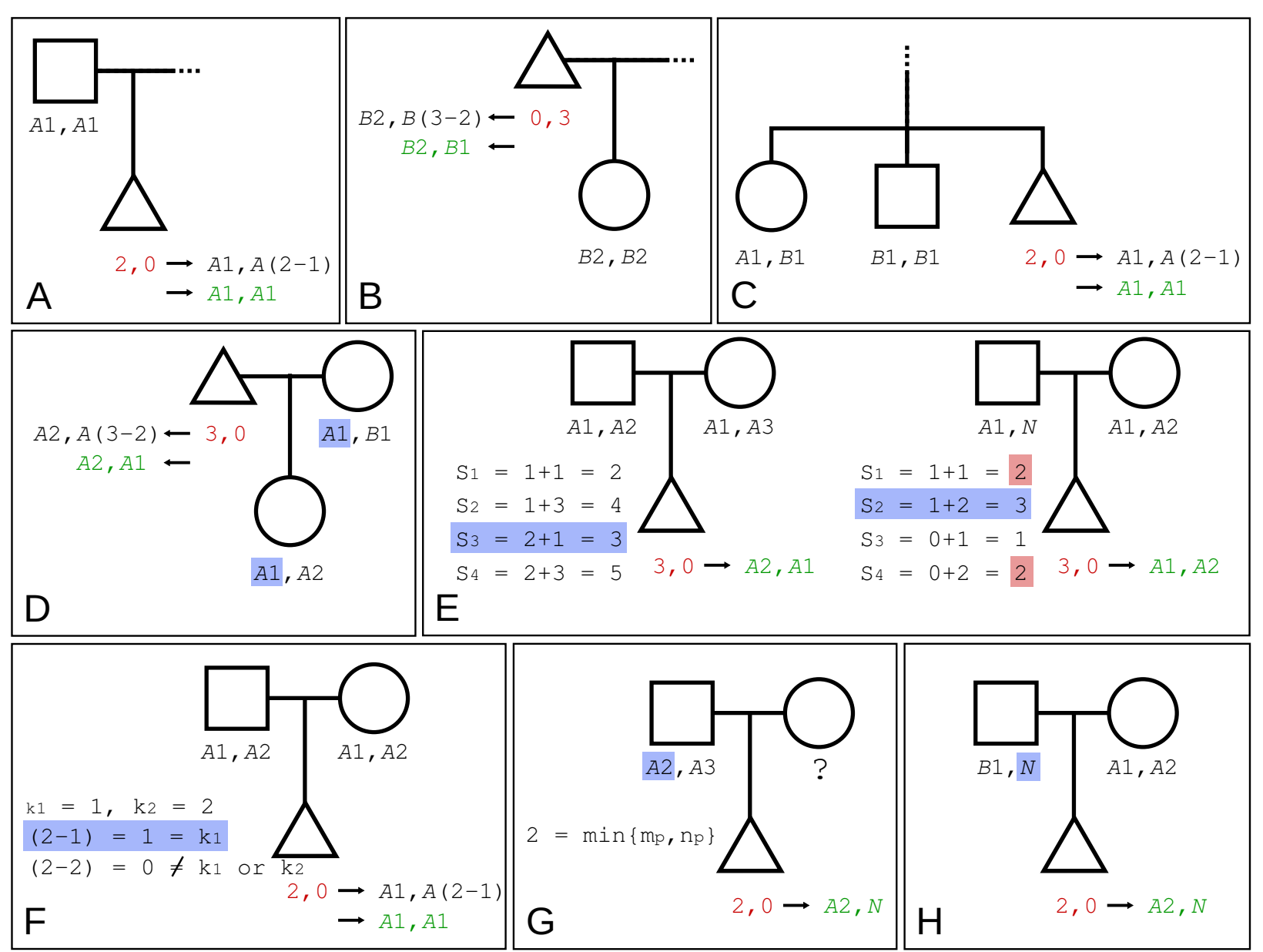

Figure 2 Type-II homozygous conversion. Representation of the conversion of Type-ll homozygous Fawkes genotypes (red) into partitioned CN genotypes (green). The triangle represents the index individual (I). (A) Conversion of /'s Fawkes genotype using one homozygous parent or (B) using one homozygous child. (C) represents the conversion using two sibs: one heterozygote and one homozygote. (D) uses a child and the spouse of the index individual. (E) uses the two homozygous parents of I. The pedigree on the left shows the conversion when there are 4 different sums, and the pedigree on the right, when there are only 3 different sums (see step 3e above). Panel (F) shows the conversion when two homozygous parents with the same genotype is used. Finally, the conversion methods when one parent has a unknown genotype or a null allele are shown in panel (G) and (H) respectively. 
this case, one $\mathrm{CN}$ allele is obligatorily shared between the child and the spouse and the remaining $\mathrm{CN}$ allele of the child can be assigned to $I$. The spouse's $\mathrm{CN}$ genotype has the form $\left[T_{1} m_{s}, T_{2} n_{s}\right]$ and the child's CN genotype $\left[T_{1} m_{c}\right.$, $\left.T_{2} n_{c}\right]$ where $\left|\left\{T_{1} m_{s}, T_{2} n_{s}\right\} \quad\left\{T_{1} m_{c}, T_{2} n_{c}\right\}\right|=1 . I$ is assigned the remaining child's $C N$ allele according to $\left\{T_{1} m_{c}, T_{2} n_{c}\right\}$ $\left\{T_{1} m_{s}, T_{2} n_{s}\right\}$ and the algorithm infers the other $\mathrm{CN}$ allele of $I$ (Figure 2D).

\section{Step $3 e$}

If the algorithm has not yet converted the Fawkes genotype according to the above steps, it then searches for cases where the two parents of $I$ are both homozygotes of the same allele type as $I$ but with distinct $\mathrm{CN}$ genotypes. Here, one parent's genotype can be inferred if its $\mathrm{CN}$ genotype is undefined. A solution exists if the first parent has a CN genotype of the form $\left[T_{1} m_{p 1}, T_{2} n_{p 1}\right]$ and the second parent, $\left[T_{1} m_{p 2}, T_{2} n_{p 2}\right]$, where $m_{p 1} \neq m_{p 2}$ or $n_{p 1} \neq n_{p 2}$. In both cases, $T_{1}=T_{2}=A$ if $I_{F, L}=[a, 0]$ and $T_{1}=T_{2}=B$ if $I_{F, L}$ $=[0, b]$. Parents may have a null allele on one chromosome. The following sums are then calculated:

$$
\begin{aligned}
& s_{1}=m_{p 1}+m_{p 2} \\
& s_{2}=m_{p 1}+n_{p 2} \\
& s_{3}=n_{p 1}+m_{p 2} \\
& s_{4}=n_{p 1}+n_{p 2}
\end{aligned}
$$

If the number of unique sums is 4 (i.e. $\left|\left\{s_{1}, s_{2}, s_{3}, s_{4}\right\}\right|=$ 4), the sum $s_{i}$ that corresponds to the Fawkes genotype of $I$ is used to assign the corresponding parental $\mathrm{CN}$ alleles to $I$. If the number of unique sums is 3 (i.e. $\left|\left\{s_{1}, s_{2}, s_{3}, s_{4}\right\}\right|=$ $3)$, then the algorithm checks whether I's Fawkes genotype matches the min or $\max \left\{s_{1}, s_{2}, s_{3}, s_{4}\right\}$, in which case the corresponding parental $\mathrm{CN}$ alleles can be assigned to $I$ (Figure 2E).

\section{Step $3 f$}

If the two homozygous parents have identical $\mathrm{CN}$ genotypes of the same allele-type as that of the index individual (first parent having a CN genotype $\left[T_{1} m_{p 1}, T_{2} n_{p 1}\right]$ and second parent, $\left[T_{1} m_{p 2}, T_{2} n_{p 2}\right]$ where $m_{p 1}=m_{p 2}=k_{1}$ and $n_{p 1}=n_{p 2}=k_{2}, T_{1}=T_{2}=A$ if $I_{F, L}=[a, 0]$ or $T_{1}=T_{2}=B$ if $I_{F}$, ${ }_{L}=[0, b]$ for both parent), then $I$ is assigned the CN genotype described in Equation (3) (Figure 2F).

$$
I_{C N, L}= \begin{cases}{\left[T_{1} k_{1}, T_{2}(a+b)-k_{1}\right]} & \text { if }\left(a+b-k_{1}\right) \\ & \in\left\{k_{1}, k_{2}\right\} \\ {\left[T_{1} k_{2}, T_{2}(a+b)-k_{2}\right]} & \text { if }\left(a+b-k_{2}\right) \\ & \in\left\{k_{1}, k_{2}\right\}\end{cases}
$$

\section{Step $3 g$}

If only one parent of $I$ has a $\mathrm{CN}$ genotype with the same $\mathrm{CN}$ allele type as $I\left(\left[T_{1} m_{p}, T_{2} n_{p}\right]\right.$ where $T_{1}=T_{2}=A$ if $I_{F, L}=$ $[a, 0]$ or $T_{1}=T_{2}=B$ if $\left.I_{F, L}=[0, b]\right)$ with the possibility of one null allele, if $(a+b)=\min \left\{m_{p}, n_{p}\right\}=z$, then $I_{C N, L}=$ $\left[T_{1} z, N\right]$ (Figure 2G).

\section{Step $3 h$}

Finally, if one parent of $I$ has a heterozygous $\mathrm{CN}$ genotype containing a $N \mathrm{CN}$ allele and the remaining allele is of a different allele-type as that of $I\left(i . e .\left[B n_{p}, N\right]\right.$ if $I_{F, L}=[a, 0]$ or $\left[A m_{p}, N\right]$ if $\left.I_{F, L}=[0, b]\right)$ and if both parents of $I$ have a $\mathrm{CN}$ genotype for this loci and the trio respects Mendelian transmission (or only one parent is converted or genotyped), the $N$ allele is assigned to $I$ and the second allele is inferred (Figure 2H).

\section{General procedure of the algorithm}

The developed algorithm reads a pedfile in linkage format containing the pedigree structures, and then opens the Fawkes output file generated by Birdsuite. Reading-in one marker of the Fawkes file at a time, CNGen begins by converting Fawkes genotypes of type 1 to 4 into $\mathrm{CN}$ genotypes as described in step 1 (see Table 1). Next, homozygous type-I calls are converted based on heterozygous first-degree relatives (step 2 and Figure 1). Any encountered Mendelian inconsistencies are reported. Unconverted type-I homozygous calls are flagged as type-II. Then, the algorithm attempts to partition the remaining type-II homozygous calls by inspection of the converted first-degree relatives of the index individual according to procedures described in step 3 (Figure 2). Following Mendelian laws, and based on first degree relatives' $\mathrm{CN}$ genotypes, obligate genotype assignments are resolved. The algorithm cycles to resolve all unconverted type-II homozygote each time it has successfully partitioned at least one call. When no more calls can be partitioned, remaining Fawkes calls and obligate Mendelian inconsistencies are set to a $\mathrm{CN}$ genotype of [$2,-2]$ and the algorithm proceeds to the following marker.

The algorithm outputs a tabulated file containing partitioned CN genotypes following the Fawkes' format. A log file is created and summary statistics of the partitioning procedures are sent to the standard output, including the percentage of each type of calls, the percentage of successful conversions and the number of Mendelian inconsistencies found during the process. CNGen does not specifically search for all Mendelian errors in the pedigrees but it reports those found during type-I and -II homozygous call conversions (step 2 and 3, respectively). The popular program PedCheck [16] can be used to systematically search for Mendelian errors, as per common linkage practice. A companion tool to interface with PedCheck was developed. 


\section{Results and Discussion Implementation}

The CNGen algorithm was implemented with the Python interpreter version 2.5.2. It was successfully tested on current Linux, Windows and Mac OS workstations. System resource requirements are dependent on the size of the input datasets, proportionally with the number of samples in the analysis. On a modern Linux workstation, the conversion of approximately 273 million calls (909,622 markers from the Affymetrix 6.0 chip for 42 pedigrees [300 individuals]) required less than $10 \mathrm{Mb}$ of RAM and a little more than one hour of computation time. CNGen is the first software to produce partitioned copy number genotype from Birdsuite's integrated SNP genotypes. Partitioned $\mathrm{CN}$ genotypes offer the valuable possibility of using copy number variation in the context of linkage studies.

\section{Validation}

We have validated the algorithm using simulations on a multi-generational pedigree consisting of 47 individuals including 14 founders (Figure 3). Gene-dropping simulations were generated. First, founders were assigned a null, hemizygote, heterozygote or homozygote $\mathrm{CN}$ genotype state following proportions given by real data $(\sim 0.0337 \%$, $\sim 2.14 \%, \sim 26.9 \%$ and $\sim 70.9 \%$, respectively). An allele is then randomly chosen from a set of all possible $\mathrm{CN}$ genotypes depending of the given state. Mendelian segregation laws were used to assign $\mathrm{CN}$ genotypes to nonfounding pedigree members, receiving one random allele from each parent. $1 \%$ of all $\mathrm{CN}$ genotypes were randomly selected and recoded as undefined $\mathrm{CN}$ genotypes ([-1, 1]). $\mathrm{CN}$ allele frequencies are presented in Table $2 . \mathrm{CN}$ genotypes were then converted into Fawkes genotypes based on the number of $A$ and $B$ alleles (i.e. $[A m, B n] \mathrm{T}$ $[m, n],[A m, A n] \mathrm{T}[(m+n), 0],[B m, N] \mathrm{T}[0, m]$, etc.). Finally, CNGen was used to partition the Fawkes genotype back into $\mathrm{CN}$ genotypes and comparison between the true $\mathrm{CN}$ genotypes and the ones inferred by CNGen
Table 2: Allele frequencies after simulation. $\mathrm{CN}$ allele frequencies after three million simulations, frequencies for 14 founders and all 47 pedigree members are presented.

\begin{tabular}{crlrl}
\hline Alleles & \multicolumn{2}{c}{ Only Founders } & \multicolumn{2}{c}{ All individuals } \\
& \multicolumn{2}{c}{$\mathbf{n}$} & $\mathbf{n}$ & $\%$ \\
\hline$A 1$ & 12294813 & 0.146 & 41275221 & 0.146 \\
$A 2$ & 10728256 & 0.128 & 35999291 & 0.128 \\
$A 3$ & 6661654 & 0.0793 & 22367164 & 0.0793 \\
$A 4$ & 4068729 & 0.0484 & 13667675 & 0.0485 \\
$A 5$ & 1472417 & 0.0175 & 4938921 & 0.0175 \\
$B 1$ & 12291382 & 0.146 & 41262843 & 0.146 \\
$B 2$ & 10732261 & 0.128 & 36029869 & 0.128 \\
$B 3$ & 6662835 & 0.0793 & 22364446 & 0.0793 \\
$B 4$ & 4068582 & 0.0484 & 13652583 & 0.0484 \\
$B 5$ & 1473733 & 0.0175 & 4949877 & 0.0176 \\
$N$ & 12706756 & 0.151 & 42672446 & 0.151 \\
-1 & 838582 & 0.00998 & 2819664 & 0.01 \\
& & & & \\
\hline Total & $\mathbf{8 4 0 0 0 0 0 0}$ & $\mathbf{2 8 2 0 0 0 0 0 0}$ \\
\hline
\end{tabular}

were compared. Three million validation runs were thus completed, for which more than 140 million genotype conversions were made, and which covered every possible conversion step from Fawkes to CN genotypes (additional file 1). The validation procedure confirmed that all converted genotypes by CNGen were accurate. Irresolvable homozygous type-II calls due to lack of information from first-degree relatives were checked and validated.

For an additional 30,000 validation runs (additional file 2), we substituted the $\mathrm{CN}$ genotype of a random pedigree member with a different randomly selected $\mathrm{CN}$ genotype and allowed $10 \%$ of $\mathrm{CN}$ genotypes to be recoded as undefined $\mathrm{CN}$ genotypes $([-1,-1])$. Following CNGen, we ran PedCheck and nuclear families where inconsistent transmissions were found were set to missing. Overall, $81 \%$ of

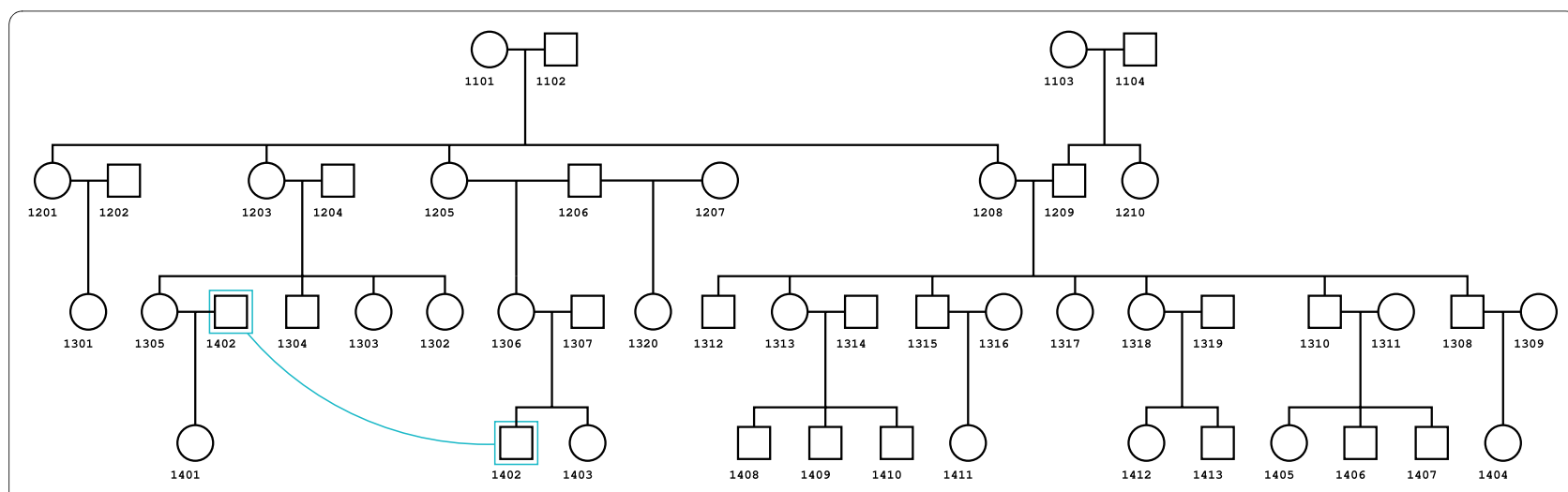

Figure 3 Complex pedigree for the simulation. Representation of the complex pedigree used for validation simulation runs. The pedigree has 47 individuals including 14 founders. Individual 1402 creates a consanguinity loop in the pedigree. The diagram is modified from Cranefoot's resulting pedigree [18]. 
the inserted $\mathrm{CN}$ genotype errors were detected by the process. 59\% of 30,000 simulations resulted in the concerned nuclear family being detected by PedCheck. In $22 \%$ of the 30,000 simulation runs, CNGen had assigned an undefined $\mathrm{CN}$ genotype at the modified individual. CNGen and PedCheck assigned an undefined value to respectively $14 \%$ and $7 \%$ of the $1,410,000$ calls for a total of $21 \%$ of undefined calls. Overall, only 5,506 out of the 30,000 simulations $(18.4 \%)$ resulted in a wrong $\mathrm{CN}$ genotype assignment to the substituted individual or to his first-degree relatives, representing an undetected genotype error rate of $\sim 0.5 \%$ for $1,410,000$ calls (30,000 simulations $\times 47$ individuals) with a simulated $2.13 \%$ genotyping error rate. In a typical study exposed to a $1 \%$ genotyping error rate, this would result in $0.2 \%$ of undetected genotype errors. These findings confirm that CNGen will not result in an excess of false calls in the presence of erroneous or de novo CNP.

\section{Conclusions}

CNGen is, to our knowledge, the first software that allows the partitioning of copy number genotypes in extended pedigrees for the purpose of linkage analysis with CNPs. CNGen is a flexible, open source Python program that can process integrated SNP genotypes from the Fawkes routine of the Birdsuite program for high-density SNP genotyping arrays. Birdsuite was developed for the Affymetrix's SNP array 5.0 and 6.0, but, as mentioned by the Birdsuite authors, the concepts and approach can be applied to any genotyping array [12] and they are planning on providing support for other high-throughput genotyping platforms, such as the Illumina $1 \mathrm{M}$.

The CNGen algorithm is not limited to the Fawkes procedure. As long as the input file format is respected, CNGen will conduct the partitioning process. For instance, results from the PennCNV software [17] could be used.

The CNGen algorithm relies upon the assumption that ancestral copy number expansions are of the same allele type on a given chromosome. In a recent publication by Hastings et al. [2], a general overview of the molecular mechanisms of change in gene copy number was presented, owing strong support for the involvement of DNA repair mechanisms which would, in great majority, be concordant with chromosome-specific expansions. There is a range of possibilities however, and copy number expansions occurring during recombination at meiosis, for example, could lead to different allele-type $\mathrm{CN}$ expansions. For regions where the assumption of identical allele-type in expansions doesn't hold, the majority will lead to Mendelian inconsistencies following the partitioning algorithm, and will be removed during data quality controls. This will result in a lower number of partitioned genotype for linkage analysis.
Our simulation experiments support the validity of the CNGen algorithm and its robustness to Fawkes genotype errors and de novo mutations.

\section{Availability and requirements \\ Project name: CNGen}

Project home page: http://www.statgen.org/ in the download section

Operating system(s): Platform independent

Programming language: Python $^{\mathrm{Tm}}$

Other requirements: Standard Python Software 2.5 or 2.6

\section{License: none}

Any restrictions to use by non-academics: none

\section{Additional material}

\begin{abstract}
Additional file 1 Archive containing the simulated data for 250 thousand runs (out of 3 million) on a pedigree containing 47 individuals ( 14 founders). The archive contains the simulated Fawkes' calls (file validation.fawkes_calls), the partitioned genotyped compute by CNGen and the corresponding log file (file

cn_genotype_calls_validation and CNGen. log, respectively) and the pedfile corresponding to the complex pedigree used for simulation (file pedfile.txt). The file (18 Mb) has been uploaded with the present document, and is also available at http://www.statgen.org/main/

index.php/Downloads/Downloads.

Additional file $\mathbf{2}$ thousand validations with errors.tar.bz2. Archive con taining the simulated data for 3 thousand runs with Mendelian errors. The archive contains the same file structure as the first additional file. The data has been splited into three files because of PedCheck's limitations. The file (2.2 Mb) has been uploaded with the present document, and is also available at http://www.statgen.org/main/index.php/Downloads/Downloads.
\end{abstract}

Authors' contributions

LPLP worked on the methodology of CNGen, implemented CNGen and the companion software, performed the validation of the algorithm using simulations and drafted the manuscript. GA participated in the validation of the algorithm and helped to draft the manuscript. GUA participated in the conception of the study and helped to draft the manuscript. MPD conceived of the study, participated in its design and coordination, produced the methodology behind CNGen and helped to draft the manuscript. All authors read and approved the final manuscript.

\section{Acknowledgements}

Funding was provided by the Canadian Institutes of Health Research (CIHR) and Heart and Stroke Foundation of Canada (GMHD 79045). MPD is supported by the FRSQ (Fonds de la recherche en santé du Québec).

\section{Author Details}

1 Montreal Heart Institute Research Center, 500, Bélanger Street, Montréal, Canada, 2 Université de Montréal, 2900, chemin de la tour, Montréal, Canada and ${ }^{3} \mathrm{CHU}$ Sainte-Justine, 3175, Chemin de la Côte-Sainte-Catherine, Montréal, Canada

Received: 3 September 2009 Accepted: 3 May 2010

Published: 3 May 2010

\section{References}

1. Beckmann JS, Estivill X, Antonarakis SE: Copy number variants and genetic traits: closer to the resolution of phenotypic to genotypic variability. Nature Reviews Genetics 2007, 8(8):639-646.

2. Hastings PJ, Lupski JR, Rosenberg SM, Ira G: Mechanisms of change in gene copy number. Nature Reviews Genetics 2009, 10:551-563. 
3. Lupski JR: Genomic disorders: structural features of the genome can lead to DNA rearrangements and human disease traits. Trends in Genetics 1998, 14:417-422

4. Stranger BE, Forrest MS, Dunning M, Ingle CE, Beazley $C$, Thorne N, Redon R, Bird CP, de Grassi A, Lee C, Tyler-Smith C, Carter N, Scherer SW, Tavaré S, Deloukas $P$, Hurles ME, Dermitzakisi ET: Relative impact of nucleotide and copy number variation on gene expression phenotypes. Science 2007, 315(5813):848-853.

5. Redon R, Ishikawa S, Fitch KR, Feuk L, Perry GH, Andrews TD, Fiegler $H$, Shapero MH, Carson AR, Chen W, Cho EK, Dallaire S, Freeman JL, Gonzalez JR, Gratacos M, Huang J, Kalaitzopoulos D, Komura D, MacDonald JR, Marshall CR, Mei R, Montgomery L, Nishimura K, Okamura K, Shen F, Somerville MJ, Tchinda J, Valsesia A, Woodwark C, Yang F, Zhang J, Zerjal T, Zhang J, Armengol L, Conrad DF, Estivill X, Tyler-Smith C, Carter NP, Aburatani H, Lee C, Jones KW, Scherer SW, Hurles ME: Global variation in copy number in the human genome. Nature 2006, 444:444-454.

6. Wong KK, deLeeuw RJ, Dosanjh NS, Kimm LR, Cheng Z, Horsman DE, MacAulay C, Ng RT, Brown CJ, Eichler EE, Lam WL: A comprehensive analysis of common copy-number variations in the human genome. The American Journal of Human Genetics 2007, 80:91-104.

7. Inoue K, Lupski JR: Molecular mechanisms for genomic disorders. Annual review of genomics and human genetics 2002, 3:199-242.

8. Clerget-Darpoux F, Elston RC: Are linkage analysis and the collection of family data dead? Prospects for family studies in the age of genomewide association. Human Heredity 2007, 64:91-96.

9. Elston RC, Lin D, Zheng G: Multistage sampling for genetic studies. Annual Review of Genomics and Human Genetics 2007, 8:327-342.

10. Nagelkerke NJD, Hoebee B, Teunis P, Kimman TG: Combining the transmission disequilibrium test and case-control methodology using generalized logistic regression. European Journal of Human Genetics 2004, 12(11):964-970.

11. Wang $T$, Elston RC: A quantitative linkage score for an association study following a linkage analysis. BMC genetics 2006, 7:5-16.

12. Korn JM, Kuruvilla FG, McCarroll SA, Wysoker A, Nemesh J, Cawley S, Hubbell E, Veitch J, Collins PJ, Darvishi K, Lee C, Nizzari MM, Gabriel SB, Purcell S, Daly MJ, Altshuler D: Integrated genotype calling and association analysis of SNPs, common copy number polymorphisms and rare CNVs. Nature Genetics 2008, 40(10):1253-1260.

13. McCarroll SA, Kuruvilla FG, Korn JM, Cawley S, Nemesh J, Wysoker A, Shapero MH, de Bakker PIW, Maller JB, Kirby A, Elliott AL, Parkin M, Hubbell E, Webster T, Mei R, Veitch J, Collins PJ, Handsaker R, Lincoln S, Nizzari M, Blume J, Jones KW, Rava R, Daly MJ, Gabriel SB, Altshuler D: Integrated detection and population-genetic analysis of SNPs and copy number variation. Nature Genetics 2008, 40(10):1166-1174.

14. Purcellb S, Neale B, Todd-Brown K, Thomas L, Ferreira MA, Bender D, Maller J, Sklar P, de Bakker PI, Daly MJ, Sham PC: PLINK: a tool set for wholegenome association and population-based linkage analyses. The American Journal of Human Genetics 2007, 81(3):559-575.

15. Abecasis GR, Cherny SS, Cookson WO, Cardon LR: Merlin--rapid analysis of dense genetic maps using sparse gene flow trees. Nature genetics 2002, 30:97-101

16. O'Connell JR, Weeks DE: PedCheck: a program for identification of genotype incompatibilities in linkage analysis. The American Journal of Human Genetics 1998, 63:259-266.

17. Kai Wang ML, Hadley D, Liu R, Glessner J, Grant SF, Hakonarson H, Bucan M: PennCNV: An integrated hidden Markov model designed for highresolution copy number variation detection in whole-genome SNP genotyping data. Genome Research 2007, 17:1665-1674.

18. Mäkinen VP, Parkkonen M, Wessman M, Groop PH, Kanninen T, Kaski K: High-throughput pedigree drawing. European Journal of Human Genetics 2005, 13:987-989.

doi: 10.1186/1471-2105-11-226

Cite this article as: Perreault et al., Partitioning of copy-number genotypes in pedigrees BMC Bioinformatics 2010, 11:226

\section{Submit your next manuscript to BioMed Centra and take full advantage of:}

- Convenient online submission

- Thorough peer review

- No space constraints or color figure charges

- Immediate publication on acceptance

- Inclusion in PubMed, CAS, Scopus and Google Scholar

- Research which is freely available for redistribution 